\title{
Tumor fibroso solitario de la pleura: Factores asociados a comportamiento maligno y recidiva. Experiencia institucional
}

\author{
Silviano Ríos-Pascual,®Juan Carlos Vázquez-Minero, Guillermo Oscar Garza-Jiménez, \\ José de Jesús López-Luna, Tania Islem-Gamboa
}

Instituto Nacional de Enfermedades Respiratorias Ismael Cosío Villegas, Ciudad de México, México.

\begin{abstract}
RESUMEN. Introducción: El tumor fibroso solitario de la pleura representa menos del $5 \%$ de los tumores pleurales primarios, de 12 a $32 \%$ pueden tener comportamiento maligno con recurrencia local y metástasis. El estudio diagnóstico de elección es la tomografía computarizada de tórax. La mayoría se origina en la pleura visceral y son pediculados. El tratamiento es la resección quirúrgica en bloque por la alta probabilidad de recurrencia, con márgenes de $1-2 \mathrm{~cm}, 9 \%$ de los casos presenta recidiva. Objetivos: Identificar factores relacionados a comportamiento maligno y recidiva. Material y métodos: Estudio observacional, descriptivo, retrospectivo y transversal. Se incluyó a todos los pacientes sometidos a resección de tumor fibroso solitario de la pleura del Instituto Nacional de Enfermedades Respiratorias Ismael Cosío Villegas, Ciudad de México, de enero 2008 a diciembre 2017. Resultados: Se incluyeron 26 pacientes, la frecuencia mayor fueron tumores pediculados dependientes de la pleura visceral, 92\% manifestó síntomas, principalmente disnea. Dieciocho benignos y ocho malignos, de los cuales dos recidivaron. Se presentó muerte asociada al tumor en dos casos. Conclusiones: Los factores relacionados a comportamiento maligno fueron la presencia de síntomas generales $(p=0.013)$, pérdida de peso mayor de $10 \mathrm{~kg}$ en los últimos seis meses $(p=0.004)$ e invasión a estructuras adyacentes $(p=0.020)$. Los factores relacionados a recurrencia fueron la presencia $>4$ mitosis $/ 10$ campos de alto poder $(p=0.018)$, pérdida de peso mayor de $10 \mathrm{~kg}$ en los últimos seis meses $(p=0.046)$, invasión a estructuras adyacentes $(p=0.030)$ y resección quirúrgica subóptima $(p=0.003)$. El tumor fibroso solitario de la pleura es un tumor de comportamiento biológico incierto, por lo que deberán investigarse factores asociados a malignidad y recurrencia. El tratamiento es la resección quirúrgica en bloque y en caso de presentarse criterios de malignidad o recidiva se requiere terapia adyuvante.
\end{abstract}

Palabras clave: Tumor fibroso solitario de la pleura, neoplasias pleurales, malignidad, recidiva.

\section{Fibrous solitary tumor of the pleura: Factors associated with malignant behavior and relapse Institutional experience}

ABSTRACT. Introduction: The fibrous lone tumor of the pleura represents less than $5 \%$ of the primary pleural tumors, from 12 to $32 \%$ can have malignant behavior with local recurrence and metastasis. The diagnostic study of choice is the computed tomography of thorax. Most originate in the visceral pleura and are pediculated. The treatment is surgical resection en bloc due to the high probability of recurrence, with margins of $1-2 \mathrm{~cm} .9 \%$ of cases have recurrence. Objectives: Identify factors related to malignant behavior and recurrence. Material and methods: Observational, descriptive, retrospective and transversal study. All patients undergoing resection of TFSP are included in the National Institute of Respiratory Diseases, from January 2008 to December 2017. Results: 26 patients are included, most frequently pediculated tumors dependent on the visceral pleura, 92\% manifested symptoms, mainly dyspnoea. Eighteen benign and eight malignant, of which two relapsed, there was death associated with the tumor in two cases. Conclusions: The factors related to malignant behavior were the presence of general symptoms $(p=0.013)$, weight loss greater than $10 \mathrm{~kg}$ in the last six months $(p=0.004)$ and invasion of adjacent structures $(p=0.020)$. The factors related to recurrence were the presence $>4$ mitoses $/ 10$ high power fields $(p=0.018)$, weight loss greater than $10 \mathrm{~kg}$ in the last six months $(p=0.046)$, invasion of adjacent structures $(p=0.030)$ and suboptimal surgical resection $(p=0.003)$. Fibrous lone tumor of the pleura is a tumor of uncertain biological behavior, therefore, factors associated with malignancy and recurrence should be investigated. The treatment is surgical resection en bloc and if malignancy or recurrence criteria are present, adjuvant therapy is required.

Key words: Fibrous solitary tumor of the pleura, pleural neoplasms, malignancy, relapse.

\section{$\triangle$ Autor para correspondencia:}

Dr. Silviano Ríos Pascual, Instituto Nacional de Enfermedades

Respiratorias Ismael Cosío Villegas, Ciudad de México, México.

Correo electrónico: silviano.rios.p@gmail.com

Trabajo recibido: 27-VIII-2018; aceptado: 18-X-2018

\section{INTRODUCCIÓN}

El tumor fibroso solitario de la pleura (TFSP) tiene una incidencia de 2.8 casos $/ 100,000$ habitantes por año, afectando con mayor frecuencia a mujeres en la sexta 

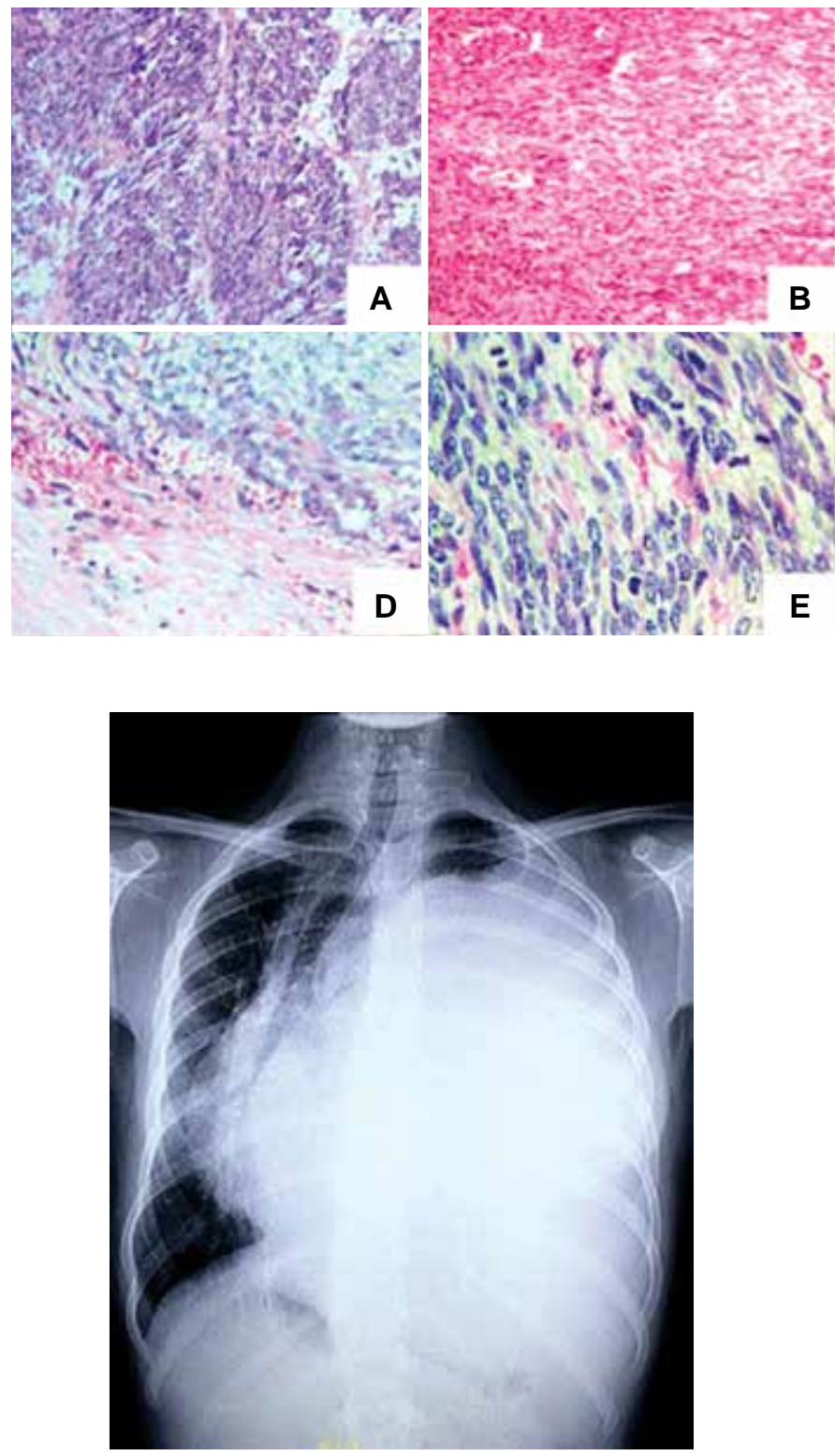

Figura 2. Radiografía posteroanterior de tórax.

y séptima décadas de la vida. ${ }^{1,2}$ La mayoría de TFSP tiene un comportamiento benigno, aunque de 12 a $32 \%$ pueden tener un comportamiento maligno llevando a la muerte por recurrencia local y metástasis. ${ }^{2}$ Además, el TFSP benigno puede transformarse a maligno incluso muchos años después de su resección completa, ${ }^{1}$ reportándose recidiva hasta 17 años después.

Histológicamente por definición es vimentina positivo, ${ }^{2}$ el CD34 y Bcl-2 se expresan en la mayoría. ${ }^{2}$ En 1989, England et al. describieron las características de malignidad como tumores de alta celularidad, pleomorfismo nuclear, $>4$ mitosis/10 campos de alto poder

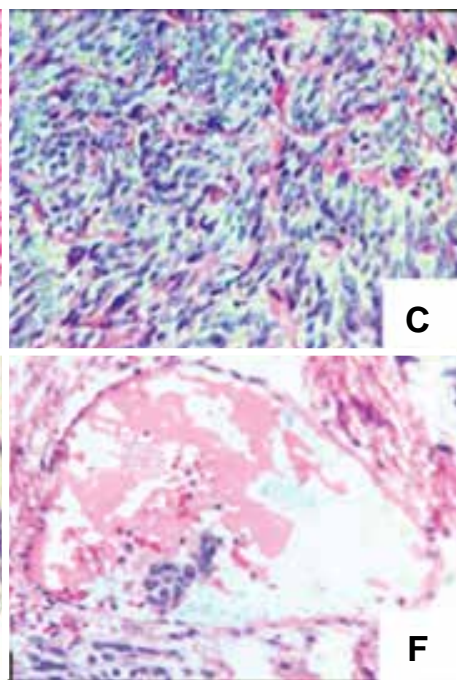

\section{Figura 1.}

A: HE áreas hiper-e hipocelulares entremezcladas con haces de colágeno y fibrosis; B: células fusiformes pleomórficas; $\mathbf{C}$ : patrón vascular hemangiopericitoide; D: infiltración a pleura parietal; E: mitosis atípicas (> 4 mit/10 CAP); F: infiltración vascular.

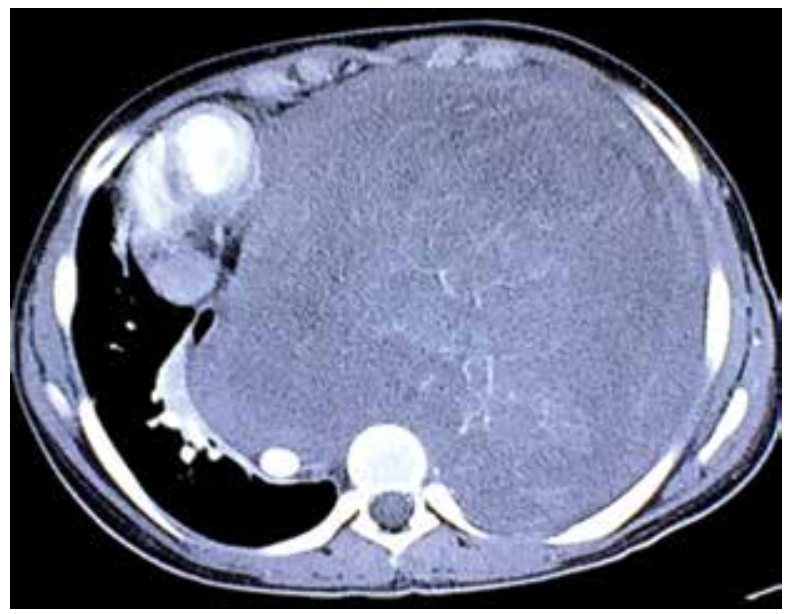

Figura 3. Tomografía computarizada de tórax.

(figura 1A-F). El TFSP maligno típicamente se presenta como neoplasias lobuladas de diámetro mayor de 10 $\mathrm{cm}$, con zonas de hemorragia y necrosis, ${ }^{3}$ la mayoría se origina en la pleura visceral (68-78\%) y son pediculados (57-83\%). ${ }^{4,5} \mathrm{Al}$ momento del diagnóstico se presentan síntomas en $50-72 \%$ de los pacientes, ${ }^{5}$ siendo los más frecuentes dolor torácico (16-59\%), disnea (14-40\%) y tos $(8-24 \%)$. 5,6

El estudio inicial es la radiografía posteroanterior de tórax (figura 2) y la tomografía computarizada de tórax es el estudio de elección ${ }^{7}$ (figura 3). El tratamiento es la resección quirúrgica en bloque por la alta probabilidad de recurrencia, con márgenes de $1-2 \mathrm{~cm} .{ }^{5,8}$ Para los tumores malignos o recidivantes se recomienda terapia adyuvante con quimio- o radioterapia. ${ }^{9}$ La tasa de recurrencia es de hasta $9 \%$, principalmente en tumores malignos y sésiles, ocasionando la muerte por tumores 
intratorácicos extensos irresecables y metástasis a distancia. ${ }^{10}$

De Perrot et al. establecen una clasificación pronóstica con cuatro categorías de acuerdo con su probabilidad de recurrencia, clasificando al TFSP en tipo 1: tumor pediculado benigno con $2 \%$ de recurrencia; tipo 2: tumor benigno sésil con $8 \%$; tipo 3: tumor maligno pediculado con $14 \%$, y tipo 4: tumor maligno sésil con $63 \%$ de recurrencia y $30 \%$ de mortalidad a dos años. ${ }^{11}$

Presentamos la experiencia en el manejo del TFSP en el Instituto Nacional de Enfermedades Respiratorias (INER) Ismael Cosío Villegas de la Ciudad de México, así como los casos de recidiva y sus características.

\section{MATERIAL Y MÉTODOS}

Estudio observacional, descriptivo, retrospectivo y transversal. Se incluyó a los pacientes sometidos a resección de TFSP en el INER de enero de 2008 a diciembre de 2017. Se describen datos sociodemográficos, presentación clínica y por imagen, manejo quirúrgico, análisis histopatológico e inmunohistoquímico del tumor y el seguimiento posquirúrgico. Se describen la tasa de recidiva y de mortalidad, describiendo la asociación entre éstas y los factores asociados a malignidad encontrados y estableciendo una relación entre los hallazgos de esta serie y lo descrito en la literatura. El análisis estadístico se realizó con el programa Epi info 3.5.1, para las variables dicotómicas se utilizó la prueba de $\chi^{2} y$, en caso de no ser adecuada, la prueba exacta de Fisher.

\section{RESULTADOS}

En el período de 2008 a 2017 se intervinieron en el INER a 26 pacientes para resección de TFSP, 17 mujeres $(65.3 \%)$ y nueve hombres (34.6\%), la edad promedio de presentación fue 52.7 años con un rango de 17 a 73 años.

Se presentaron síntomas en 24 pacientes (92.3\%), los más frecuentes fueron: disnea en 18 pacientes (69.2\%); tos en 16 (61.5\%); dolor torácico en 12 (46.1\%); síntomas generales (astenia, adinamia, fiebre, diaforesis) en siete (26.9\%); pérdida de peso $>10 \mathrm{~kg}$ en seis (23\%); síndromes paraneoplásicos en un paciente que presentó Doege-Potter (3.8\%).

El tamaño promedio fue de $19 \mathrm{~cm}$ con un rango de 32 a $2 \mathrm{~cm}$, de los cuales $21(80.7 \%)$ fueron mayores de $10 \mathrm{~cm}$. Se encontró pedículo en 17 casos $(65.3 \%)$ y derrame pleural en 11 (42.3\%).

La distribución topográfica del tumor fue basal izquierda en 14 casos (53.8\%), basal derecha en seis (23\%), apical en cuatro (15.3\%) e intraparenquimatoso en dos (7.6\%). Se presentó invasión a estructuras adyacentes en cinco casos (19.2\%) y desplazamiento mediastinal en 13 (50\%). El origen de los tumores fue de pleura visceral en 17 pacientes (65.3\%), 11 en TFSP benignos (61.1\%) y sies en TFSP malignos (75\%).

En cuanto al abordaje quirúrgico, éste fue toracotomía posterolateral en 19 pacientes (73\%) (figura 4) con tumores de entre 8 a $30.5 \mathrm{~cm}$, Clamshell en tres pacientes $(11.5 \%)$ con tumores de 27 a $32 \mathrm{~cm}$, y toracoscopia en cuatro pacientes (15.3\%) para tumores de 2 a $5 \mathrm{~cm}$. Se requirió resección extendida en 17 pacientes (65.3\%) (figura 5): resección pulmonar en cuña en 14 pacientes

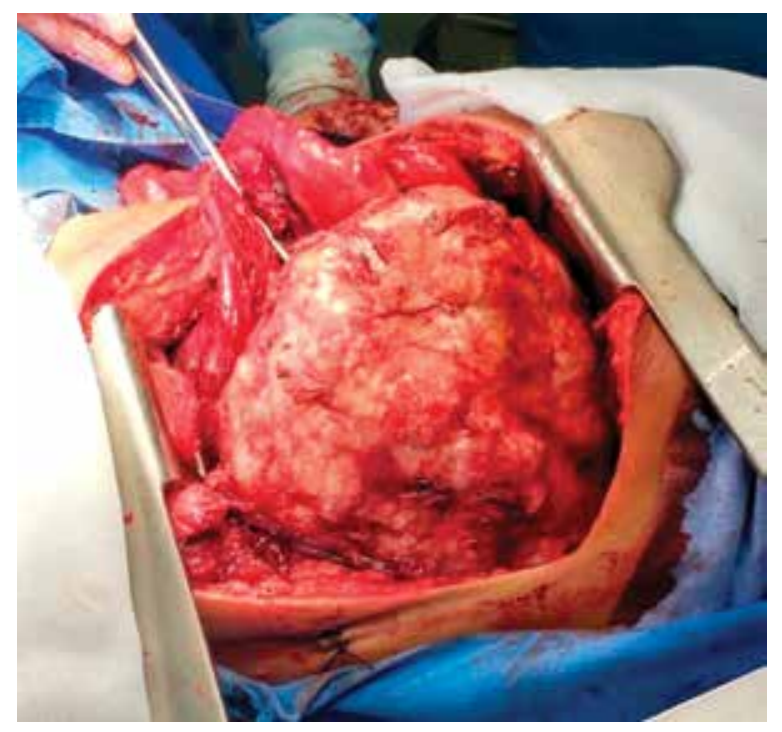

Figura 4. Toracotomía posterolateral.

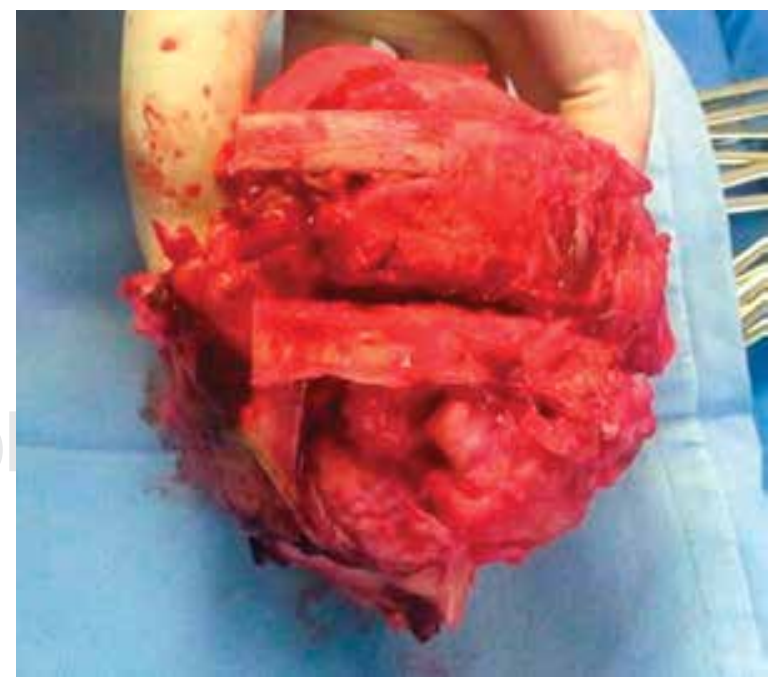

Figura 5. Resección extendida incluyendo dos arcos costales y músculo intercostal. 
(53.8\%), lobectomía en uno (3.8\%), neumonectomía en uno (3.8\%) y resección de tumor en bloque con pared torácica en un caso (3.8\%) (figura 6). El sangrado transoperatorio promedio fue de $998 \mathrm{ml}$ con el mayor sangrado de $5 \mathrm{I}$ durante la resección de tumor de 30.5 $\mathrm{cm}$ en eje mayor, el cual requirió neumonectomía. Se presentaron complicaciones posquirúrgicas en seis pacientes $(23 \%)$ : dolor posquirúrgico $>30$ días en tres pacientes $(11.5 \%)$, alteraciones del ritmo cardíaco en dos pacientes (7.69\%), neumonía en un paciente (3.8\%), infección de herida quirúrgica en dos pacientes (7.69\%). La mortalidad perioperatoria fue $11.5 \%$ (tres pacientes) secundario a choque hipovolémico (un caso), choque cardiogénico posterior a descompresión cardíaca (un caso) en un tumor de $32 \times 24 \times 17 \mathrm{~cm}$ con peso aproximado de $5 \mathrm{~kg}$ y un caso secundario a deterioro ventilatorio y alteraciones del ritmo cardíaco por compresión tumoral.

El resultado histopatológico de la pieza quirúrgica fue TFSP en todos los casos, 18 benignos (69.2\%) y ocho malignos (30.7\%) de acuerdo con criterios establecidos previamente. La inmunohistoquímica de estos tumores resultó positiva para CD34 en 25 casos (96.1\%), vimentina en 20 (76.9\%), Bcl-2 en 11 (42.3\%) y CD99 en siete (26.9\%). De los ocho casos catalogados como malignos, se presentó recidiva en dos (25\%) (tabla 1). Los factores correspondientes a malignidad fueron $>4$ mitosis/10 campos de alto poder, hipercelularidad, pleomorfismo nuclear, Ki67 > 5\% y necrosis $>10 \%$ (tabla 2 ).

El primer caso de recidiva fue en mujer de 63 años con un tumor basal izquierdo que ocupaba $90 \%$ de

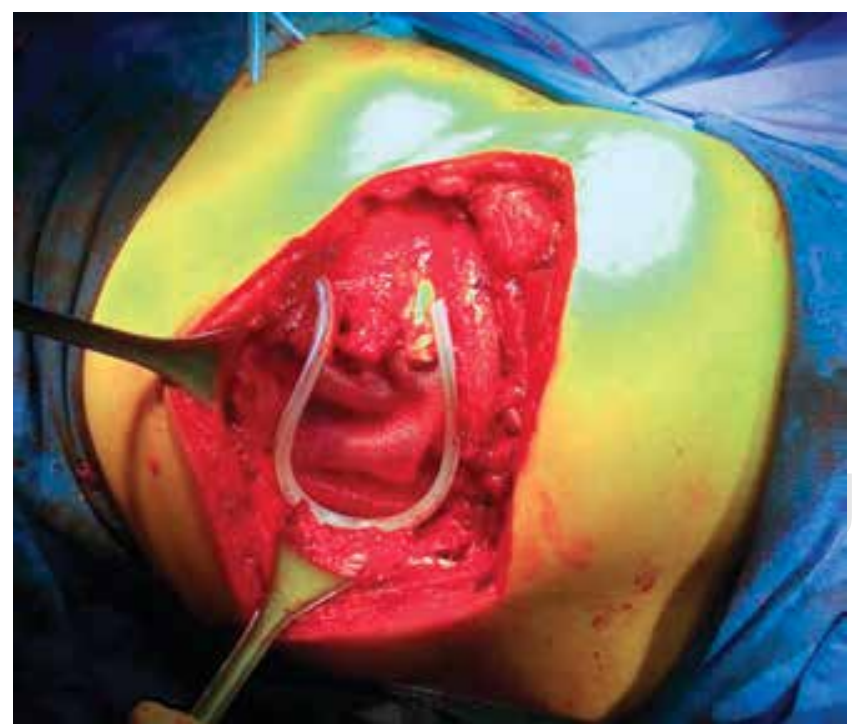

Figura 6. Resección de pared torácica y reconstrucción con malla de polipropileno. hemitórax, se realizó tumorectomía parcial con reporte histopatológico de TFSP benigno, adecuada evolución posquirúrgica. Después de 14 meses presentó recidiva, resecando tumor bilobulado de 29 y $16.3 \mathrm{~cm}$, pediculado a pleura visceral con invasión a pared torácica, requiriendo resección extendida de dos costillas y músculos intercostales, con sangrado transoperatorio de 2,100 $\mathrm{ml}$. El reporte histopatológico de la pieza quirúrgica fue compatible con TFSP maligno, la paciente evolucionó al deterioro de la función cardíaca con alteraciones persistentes del ritmo, desarrolló neumonía que evolucionó a sepsis falleciendo 25 días posteriores a la cirugía.

El segundo caso es una mujer de 73 años que fue intervenida quirúrgicamente resecando tumor con eje mayor de $25.5 \mathrm{~cm}$, requiriendo resección pulmonar en cuña (lóbulo medio y superior), con resección subóptima de lesión en superficie diafragmática, se evacuaron $150 \mathrm{ml}$ de derrame pleural, sangrado transoperatorio de 2,800 ml. El reporte histopatológico de la pieza quirúrgica reportó TFSP con hipercelularidad, $>10$ mitosis atípicas/10 campos de alto poder, inmunohistoquímica positiva para vimentina y CD34, Ki67:1 5\%. En el mes, 23 pacientes de seguimiento posquirúrgico presentaron recidiva. Se resecó un tumor bilobulado de $30 \mathrm{~cm}$ en eje mayor (figura 7) adherido firmemente a diafragma

Tabla 1. Inmunohistoquímica.

\begin{tabular}{|l|r|r|l|}
\hline & \multicolumn{1}{|c|}{$\begin{array}{c}\text { Total } \\
(n=26)\end{array}$} & \multicolumn{1}{c|}{$\begin{array}{c}\text { Benignos } \\
(\mathrm{n}=18)\end{array}$} & $\begin{array}{c}\text { Malignos } \\
(\mathrm{n}=8)\end{array}$ \\
\hline Vimentina & $20(76.9 \%)$ & $13(72.2 \%)$ & $7(87.5 \%)$ \\
\hline CD34 & $25(96.1 \%)$ & $17(94.4 \%)$ & $8(100 \%)$ \\
\hline CD99 & $7(26.9 \%)$ & $4(22.2 \%)$ & $3(37.5 \%)$ \\
\hline Bcl-2 & $11(42.3 \%)$ & $7(38.8 \%)$ & $4(50 \%)$ \\
\hline
\end{tabular}

Tabla 2. Datos de malignidad y asociación a recidiva.

\begin{tabular}{|l|c|c|c|}
\hline & $\begin{array}{c}\text { Total } \\
(\mathrm{n}=8)\end{array}$ & $\begin{array}{c}\text { Recidiva } \\
(\mathrm{n}=2)\end{array}$ & \\
\hline $\begin{array}{l}>4 \text { mito- } \\
\text { sis/10 CAP }\end{array}$ & $4(50 \%)$ & $2(100 \%)$ & $\mathrm{p}=0.018$ \\
\hline $\begin{array}{l}\text { Hipercelula- } \\
\text { ridad }\end{array}$ & $5(62.5 \%)$ & $1(50 \%)$ & $\mathrm{p}=0.353$ \\
\hline $\begin{array}{l}\text { Pleomorfis- } \\
\text { mo nuclear }\end{array}$ & $2(25 \%)$ & $1(50 \%)$ & $\mathrm{p}=0.150$ \\
\hline Ki67 $>5 \%$ & $3(37.5 \%)$ & $1(50 \%)$ & $\mathrm{p}=0.221$ \\
\hline $\begin{array}{l}\text { Necrosis }> \\
10 \%\end{array}$ & $5(62.5 \%)$ & $1(50 \%)$ & $\mathrm{p}=0.457$ \\
\hline
\end{tabular}




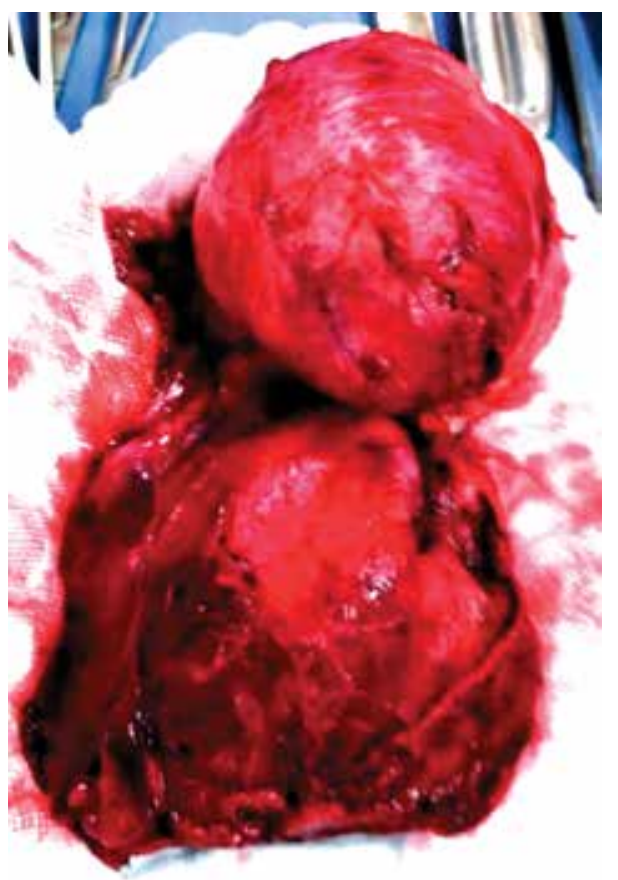

Figura 7. Pieza quirúrgica, recidiva de TFSP.

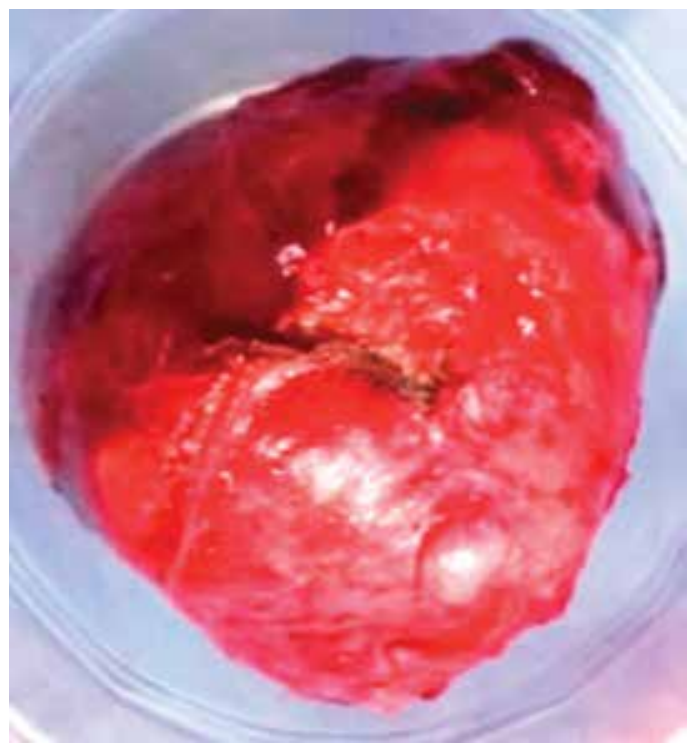

Figura 8. Pieza quirúrgica, metástasis pleural apical de TFSP.

y segmento posterior; así como una segunda lesión en topografía apical de $5 \times 8 \mathrm{~cm}$, se realiza resección de tumor en bloque y de metástasis apical (figura 8) requiriendo resección pulmonar en cuña, ambas lesiones de morfología sésil, dependientes de pleura parietal, sangrado transoperatorio de $1,800 \mathrm{ml}$. El reporte histopatológico fue compatible con TFSP maligno con pleomorfismo nuclear, hipercelularidad, $>4$ mitosis

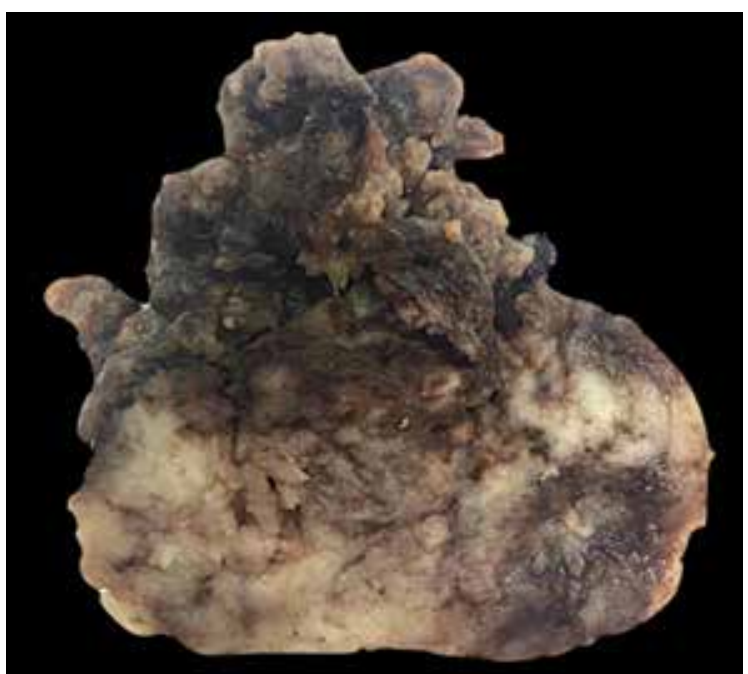

Figura 9. Fotografía macroscópica al corte de recidiva de TFSP.

atípicas/10 campos de alto poder, zonas de hemorragia y necrosis (figura 9). La inmunohistoquímica resultó positiva para vimentina, CD34, calponina y S-100. La paciente presentó un postquirúrgico normoevolutivo y se decidió terapia adyuvante con radioterapia.

\section{DISCUSIÓN}

EI TFSP es una neoplasia infrecuente, de comportamiento biológico incierto, la mayoría de TFSP se origina en la pleura visceral ${ }^{3,5}$ y son pediculados, ${ }^{1,3,6}$ lo cual concuerda con los hallazgos de nuestra serie presentándose en $65.3 \%$ de los casos. Comúnmente, es descrito como un tumor de comportamiento benigno, ${ }^{2}$ aunque en nuestra serie encontramos características de malignidad en $30.7 \%$ de los casos y recidiva en $7.7 \%$, lo cual concuerda con lo reportado por England y de Perrot et al.,411

El comportamiento biológico incierto de este tumor genera la necesidad de identificar factores asociados a comportamiento maligno y recurrencia. En el estudio de factores asociados de comportamiento maligno, Lanhon et al. ${ }^{6}$ reportaron asociación estadísticamente significativa en tumores que condicionan síntomas, sésiles y multifocales.

En nuestro estudio encontramos mayor incidencia de comportamiento maligno en tumores mayores de $10 \mathrm{~cm}$, sintomáticos y asociados a derrame pleural; sin embargo, en el análisis univariado sólo se encontró significancia estadística en la presencia de síntomas generales como fiebre, diaforesis, astenia y adinamia $(p=0.013)$, pérdida de peso mayor de $10 \mathrm{~kg}$ en los últimos seis meses $(p=0.004)$ e invasión a estructuras adyacentes $(p=0.020)$ (tabla 3$)$. 
Tabla 3. Factores asociados a malignidad.

\begin{tabular}{|l|l|}
\hline Síntomas atípicos: & $p=0.0138$ \\
\hline Pérdida de peso: & $p=0.004$ \\
\hline Invasión a estructuras adyacentes: & $p=0.020$ \\
\hline
\end{tabular}

Tabla 4. Factores asociados a recurrencia.

\begin{tabular}{|l|l|}
\hline$>4$ mitosis/10 campos de alto poder: & $p=0.018$ \\
\hline Resección subóptima: & $p=0.003$ \\
\hline Pérdida de peso: & $p=0.046$ \\
\hline Invasión a estructuras adyacentes: & $p=0.030$ \\
\hline
\end{tabular}

Es conveniente destacar que la morfología del tumor (sésil/pediculado) o el origen en pleura parietal o visceral no resultó útil para predecir el comportamiento del tumor, a diferencia de lo establecido por Tapias et al. en su sistema de clasificación pronóstica. ${ }^{8}$

El estudio de factores asociados a recurrencia ha presentado gran controversia, los principales factores asociados a recurrencia reportados por England y de Perrot et al., ${ }^{411}$ son la presencia de tumores sésiles y malignos. Boddaert et al. ${ }^{3}$ agregaron el derrame pleural y Lanhon et al. ${ }^{6}$ la negatividad para CD34. Sin embargo, en nuestra serie no se encuentra relación estadísticamente significativa entre recurrencia y la morfología sésil, derrame pleural o la negatividad para CD34, incluso el $100 \%$ de los casos de recurrencia fueron CD34 positivos. Posteriormente, Franzen et al. ${ }^{1}$ presentaron mediante análisis univariado asociación de recurrencia a tumores mayores de $10 \mathrm{~cm}$ con alto índice mitótico (> 10 mitosis atípicas en 10 campos de alto poder) y $\mathrm{Ki}-67>12 \%$.

En nuestra serie mediante análisis univariado se identifica relación estadísticamente significativa para recurrencia a la presencia de $>4$ mitosis/10 campos de alto poder $(p=0.018)$, pérdida de peso mayor de 10 $\mathrm{kg}$ en los últimos seis meses $(p=0.046)$, invasión a estructuras adyacentes $(p=0.030)$ y resección quirúrgica subóptima $(p=0.003)$ (tabla 4$)$. Ningún parámetro de inmunohistoquímica resultó estadísticamente significativo para predecir comportamiento maligno o recurrencia. Debido al número limitado de la muestra no es posible realizar un análisis multivariado.

\section{CONCLUSIONES}

El TFSP es una neoplasia infrecuente, cuando son mayores de $15 \mathrm{~cm}$ tienen mayor probabilidad de pre- sentar comportamiento maligno y los mayores de 25 $\mathrm{cm}$ tienen mayor riesgo de recurrencia $(p=0.06)$. Aunque el tratamiento estándar es la resección en bloque del tumor con estructuras adyacentes involucradas. El $30 \%$ de los tumores resecados presenta características de malignidad, principalmente en tumores mayores de $10 \mathrm{~cm}(p=0.13)$, que se acompañan de síntomas atípicos $(p=0.004)$ e invasión a estructuras adyacentes $(p=0.02)$. En estos tumores se recomienda terapia adyuvante con quimio- o radioterapia. Se presenta recidiva en $7.7 \%$ de los casos durante los primeros 24 meses posteriores a la resección quirúrgica. Por ello, se recomienda seguimiento con tomografía computarizada de tórax cada seis meses durante el primer año y posteriormente en forma anual. Los principales factores asociados a recidiva son la resección quirúrgica subóptima $(p=0.03)$ y presencia en la pieza quirúrgica de más de 4 mitosis/10 campos de alto poder $(p=0.01)$. El tratamiento de la recidiva es resección quirúrgica en bloque acompañada de terapia adyuvante.

\section{Conflicto de intereses}

Los autores declaran no tener conflicto de intereses.

\section{REFERENCIAS}

1. Franzen D, Diebold M, Soltermann A, et al. Determinants of outcome of solitary fibrous tumors of the pleura: an observational cohort study. BMC Pul Med 2014;14:138142. doi: 10.1186/1471-2466-14-138.

2. Abu Arab W. Solitary fibrous tumours of the pleura. Eur J Cardiothoracic Surg 2012;41(3):587-597. doi: 10.1093/ ejcts/ezr009.

3. Boddaert G, Guiraudet P, Grand B, et al. Solitary fibrous tumors of the pleura: a poorly defined malignacy profile. Ann Thorac Surg 2015;99(3):1025-1031. doi: 10.1016/j.athoracsur.2014.10.035.

4. England DM, Hochholzer L, McCarthy MJ. Localized benign and malignant fibrous tumors of the pleura. A clinicopathologic review of 223 cases. Am J Surg Pathol 1989;13(8):640-658.

5. Schmid S, Csanadi A, Kaifi JT, et al. Prognostic factors in solitary fibrous tumors of the pleura. J Surg Res 2015;195(2):580-587. doi: 10.1016/j.jss.2015.01.049.

6. Lanhon B, Mercier O, Fadel E, et al. Solitary fibrous tumor of the pleura: outcomes of 157 complete resections in a single center. Ann Thorac Surg 2012;94(2):394-400. doi: 10.1016/j.athoracsur.2012.04.028.

7. Cardinale L, Ardissone F, Garetto I, et al. Imaging of benign solitary fibrous tumor of the pleura: a pictorial essay. Rare Tumors 2010;2(1):e1. doi: 10.4081/rt.2010. e1.

8. Tapias LF, Mercier O, Ghigna MR, et al. Validation of a scoring system to predict recurrence of resected solitary 
fibrous tumors of the pleura. Chest 2015;147(1):216-223. doi: 10.1378/chest.14-1180.

9. Liu M, Liu B, Dong L, Liu B. Recurrent intrathoracic solitary fibrous tumor: Remarkable response to radiotherapy. Ann Thoracic Med 2014;9(4): 245-247. doi: 10.4103/1817-1737.140142.
10. Yokoi T, Tsuzuki T, Yatabe $Y$, et al. Solitary fibrous tumour: significance of $p 53$ and CD34 inmunoreactivity in its malignant transformation. Histopathology 1998;32(5):423-432.

11. de Perrot M, Fischer S, Bründler MA, Sekine Y, Keshavjee S. Solitary fibrous tumors of the Ileura. Ann Thorac Surg 2002;74(1):285-293. 